\title{
Die Große Furcht und die Anfänge des Kalten Krieges
}

Als der Zweite Weltkrieg 1945 zu Ende ging, standen die Zeichen zunächst keineswegs auf Konfrontation. Gewiss waren die beiden Hauptsieger des Krieges mit gegensätzlichen Ordnungsvorstellungen angetreten - die amerikanische Demokratie mit der Hoffnung auf Durchsetzung liberaldemokratischer Ordnungsformen in den befreiten Ländern; der sowjetische Diktator Josef W. Stalin mit dem Anspruch, an der Spitze einer revolutionären Bewegung zu stehen, die die historisch notwendige Überwindung bürgerlich-kapitalistischer Ordnungsverhältnisse durchsetzen würde. Ihre fundamental verschiedenen Gesellschaftssysteme legten gegensätzliche außenpolitische Strategien nahe - in den USA die Ausdehnung des Freihandelsprinzips und die lautstarke Propagierung freiheitlicher Ideale; in der Sowjetunion die Abschirmung gegen kapitalistische Mächte und freiheitliche Tendenzen. Weder die USA noch die Sowjetunion verfügten über langjährige Erfahrung im Umgang mit fremden Mächten und bei beiden spielte die ideologische Betrachtungsweise internationaler Probleme traditionell eine große Rolle - das erschwerte die Verständigung zusätzlich.

Auf der anderen Seite sprachen aber auch gewichtige Gründe für eine Fortsetzung der friedlichen Zusammenarbeit, die zum Sieg der Anti-Hitler-Koalition geführt hatte, und damit für eine einvernehmliche Regelung der Friedensordnung. Weder die Führungsgruppen der USA noch die Führer der Sowjetunion wollten einen neuen Krieg. Die einen nicht, weil von allen Kosten und Leiden abgesehen die eigene Bevölkerung schon für den gerade überstandenen Krieg nur mit äußerster Mühe gewonnen worden war und das Drängen auf Demobilisierung das gesamte öffentliche Leben bei Kriegsende beherrschte. Die anderen nicht, weil ihr Regime in diesem Krieg nur knapp am Zusammenbruch vorbeimanövriert war und allein zur Wiederherstellung der Vorkriegsverhältnisse viele Jahre angestrengter Wiederaufbauarbeit benötigte. Auch wuchsen mit dem technologischen Fortschritt Kosten und Zerstörungskraft großer Kriege und erschien es schon darum geboten, sie zu vermeiden - erst recht, wenn dabei, wie man sich nun all- 
mählich bewusst wurde, eine so gefährliche Waffe wie die Atombombe zum Einsatz kommen konnte. Wenn aber Krieg als Mittel zur Gestaltung der amerikanisch-sowjetischen Beziehungen nicht in Frage kam, dann war es ein Gebot der Vernunft, das Konfliktpotential niedrig zu halten. Das wiederum legte es nahe, ein antagonistisches Nebeneinander von zwei Machtblöcken erst gar nicht entstehen zu lassen.

Trotz ihrer Gegensätze strebten die Führer der beiden Hauptsiegermächte daher zunächst eine einvernehmliche Regelung der Friedensordnung nach dem Zweiten Weltkrieg an. Stalin wollte die Einflusssphären in Europa und Asien sichern, die er militärisch errungen hatte; eine weitere Expansion des Kommunismus sollte warten, bis die gesellschaftliche Entwicklung in den westlichen Ländern dafür "reif» sein würde. Der amerikanische Präsident Franklin D. Roosevelt war davon überzeugt, dass der Frieden nur zu sichern war, wenn man die militärischen Gewinne der Sowjetunion anerkannte. In den Konferenzen von Jalta (Februar 1945) und Potsdam (Juli/August 1945) verständigten sich die sowjetische und die amerikanische Führung auf die Grundzüge einer kooperativen Nachkriegsordnung. Dazu gehörten die gemeinsame Besetzung des besiegten Großdeutschen Reiches, die Übernahme besonderer Verantwortung in den Vereinten Nationen (als permanente Mitglieder des Sicherheitsrates, zusammen mit Großbritannien, Frankreich und China) sowie eine stillschweigende Übereinkunft über besondere Interessengebiete in Europa. ${ }^{1}$

Warum haben die Vereinbarungen von Jalta und Potsdam die Spaltung Europas und den Kalten Krieg nicht verhindern können? Hier muss man auf das Wiederaufleben der "Großen Furcht» vor der bolschewistischen Revolution verweisen, die alsbald in der amerikanischen Führung aufkam und dann im westlichen Europa eine viel existenziellere Dimension annehmen sollte. Parallel dazu steigerte sich aber auch das krankhafte Misstrauen Stalins bisweilen zu einer abgrundtiefen Furcht vor einer Aggression der amerikanischen »Imperialisten«, die eine Verständigung über fortdauernde gemeinsame Interessen der beiden Siegermächte zusehends erschwerte. Damit kam eine Spirale wechselseitiger Expansionsfurcht in Gang, die in den Kalten Krieg und die Spaltung Deutschlands und Europas mündete. ${ }^{2}$

1 Vgl. im Detail Loth, Wilfried: Die deutsche Frage und der Wandel des internationalen Systems (Das Deutsche Reich und der Zweite Weltkrieg, Bd 10/2), München 2008, S. 201-378.

2 Grundlegend hierzu Loth, Wilfried: Die Teilung der Welt. Geschichte des Kalten Krieges 19411955, erweiterte Neuausgabe, München 2002. 


\section{Das Konzept der »Eindämmung»}

Das Vertrauen in die Möglichkeit langfristiger Kooperation mit der Sowjetunion wurde durch die Maßnahmen zur Sicherung ihrer Einflusssphäre in der Osthälfte Europas erschüttert: Einsetzung kommunistisch kontrollierter Regierungen in Rumänien, Bulgarien und Polen, zunehmender Druck der Kommunisten auf die Partei der kleinen Landwirte in Ungarn, die den Ministerpräsidenten stellte, schließlich die Kampagne zur Vereinigung der Sozialdemokraten mit den Kommunisten in der sowjetischen Besatzungszone Deutschlands - all das machte klar, dass die Verpflichtung auf einen »demokratischen Weg“ bei der Etablierung der Nachkriegsordnung in den befreiten Ländern, zu der sich Stalin in Jalta bereitgefunden hatte, ${ }^{3}$ das Papier nicht wert war, auf dem sie festgehalten worden war. In der Irritation, die dieses Vorgehen in der Administration von Präsident Harry S. Truman auslöste, wirkte ein langes Telegramm orientierend, mit dem George F. Kennan, damals amerikanischer Botschaftsrat in Moskau, seinen Vorgesetzten in Washington eine Erklärung lieferte.

Kennans Telegramm, das am 22. Februar 1946 in Washington einlief und schnell in allen Abteilungen der Administration verbreitet wurde, beschrieb die sowjetische Außenpolitik, das bekanntgewordene Misstrauen und die Aggressivität der Sowjetführung nicht als Folge einer aktuellen politischen Entwicklung, sondern als notwendigen Bestandteil des sowjetischen Systems.

„Die Erfordernisse ihrer eigenen vergangenen und gegenwärtigen Position sind es, die die sowjetische Führung dazu zwingen, ein Dogma zu verkünden, nach dem die Außenwelt böse, feindselig und drohend, aber zugleich von einer schleichenden Krankheit befallen und dazu verurteilt ist, von immer stärker werdenden inneren Kämpfen zerrissen zu werden, bis sie schließlich von der erstarkenden Macht des Sozialismus den Gnadenstoß erhält und einer neuen und besseren Welt weicht. Diese These liefert den Vorwand für das Anwachsen von Militär und Polizei im russischen Staat, für die Isolierung der russischen Bevölkerung von der Außenwelt und für die ständigen Versuche, die russische Polizeigewalt noch mehr auszuweiten, alles Dinge, die seit je den natürlichen Instinkten russischer Herrscher entsprechen. ${ }^{4}$

In Kennans Sicht galt das Dogma von der feindseligen kapitalistischen Umwelt unabhängig von den tatsächlichen Erfahrungen der Sowjetführung (die doch, wie er meinte, das Gegenteil nahelegen mussten); folglich war es auch durch noch so große Kooperationsbereitschaft der USA nicht möglich, der Sowjetpolitik ihre Aggressivität zu nehmen. "Alles in allem haben wir es mit einer politischen Kraft zu

3 "Gemeinsame Erklärung über das befreite Europa», in: Europa-Archiv 1 (1946), S. 211-212, hier S. 212.

4 Deutsche Übersetzung in: Kennan, George F.: Memoiren eines Diplomaten, München 1971, S. 552 568, hier S. $556 f$. 
tun, die sich fanatisch zu dem Glauben bekennt, dass es mit Amerika keinen dauerhaften Modus Vivendi geben kann, dass es wünschenswert und notwendig ist, die innere Harmonie unserer Gesellschaft, unsere traditionellen Lebensgewohnheiten und das internationale Ansehen unseres Staates zu zerstören, um der Sowjetmacht Sicherheit zu verschaffen." Die Sowjets würden alles tun, um das sozialistische Lager zu stärken und zugleich die kapitalistischen Nationen zu schwächen und untereinander aufzuhetzen. Mit Hilfe der kommunistischen Parteien, gelenkt von einem »Untergrundgeneralstab des Weltkommunismus», einer "heimlichen, von Moskau straff koordinierten und dirigierten Komintern«, würden sie versuchen, „die allgemeine strategische und politische Potenz der stärkeren Westmächte auszuhöhlen«, würden Druck ausüben, um Regierungen, die »den sowjetischen Bestrebungen im Wege sind«, von der Türkei über die Schweiz bis zu England, aus dem Amt zu entfernen, würden sie »im Ausland [...] in der Regel auf die Zerstörung aller Formen der persönlichen Unabhängigkeit, der wirtschaftlichen, politischen und moralischen, hinarbeiten. ${ }^{5}$

Als Gegenmittel forderte Kennan eine Politik der Stärke und der Sammlung der westlichen Nationen unter amerikanischer Führung: Der Erfolg der sowjetischen Politik hänge "von dem Maß an Zusammenhalt, Festigkeit und Kraft ab, die die westliche Welt aufbringen kann. "Und: „Viele fremde Völker zum mindesten in Europa, sind durch die erlittenen Erfahrungen ermüdet und verschreckt und interessieren sich weniger für abstrakte Freiheit als für Sicherheit. Sie suchen Führung eher als Verantwortung. Wir sollten besser befähigt sein als die Russen, sie ihnen zu geben. ${ }^{6}$

Die Wahrnehmung sowjetischer Politik als destruktiv und gefährlich konkretisierte sich im Winter 1946/47, als die Kriegszerstörungen in Europa zu einer gewaltigen Produktions- und Investitionskrise führten. Die Industrieproduktion hatte in Frankreich, Belgien und den Niederlanden Ende 1946 etwa 85 Prozent des Vorkriegsstands erreicht, in Italien 60 Prozent; in Großbritannien und Skandinavien hatte sie den Vorkriegsstand etwas überschritten; in Deutschland stagnierte sie, die übrigen Länder lähmend, bei etwa 36 Prozent des Standes von 1936. Zusammen genommen belief sich der Produktionsindex der sechzehn späteren Teilnehmerländer des Marshall-Plans auf 83 Prozent des Vorkriegsstandes. 1947 erzielten sie ein Zahlungsbilanzdefizit von 7,5 Milliarden Dollar, während die USA im gleichen Jahr einen Überschuss von 10 Milliarden Dollar erwirtschaf- 
teten. ${ }^{7}$ Der Mangel an Nahrungsmitteln nach einer klimatisch bedingten schlechten Ernte 1946, Kohlemangel im extrem kalten Winter 1946/47 und Transportschwierigkeiten im kriegszerstörten Deutschland verschärften den Eindruck einer hereinbrechenden Krise, die nach allem, was die europäischen Länder im Kriege an Zerstörungen erlitten hatten, noch schlimmere Auswirkungen haben musste als die Weltwirtschaftskrise nach dem »Schwarzen Freitag“ vom Oktober 1929.

Diese Krise, darin waren sich alle amerikanischen Beobachter im Frühjahr 1947 einig, galt es mit allen Mitteln zu vermeiden - nicht nur, weil die akute Notlage die europäischen Regierungen wieder zu protektionistischen Maßnahmen trieb und so die amerikanische Open-door-Politik in Gefahr geriet und auch nicht nur, weil ein Zusammenbruch Europas den USA den wichtigsten Handels- und Absatzmarkt nehmen und so eine weltweite Rezession auslösen würde. Noch bedrohlicher schien, dass diese Krise von den kommunistischen Bewegungen in den verschiedenen europäischen Ländern ausgenutzt werden konnte, um im Interesse Moskaus die Macht an sich zu reißen. In der Sicht der amerikanischen Planer fügten sich so die Erinnerungen an die Weltwirtschaftskrise und die Dogmen der Eindämmungsdoktrin zu einem geradezu apokalyptischen Bild zusammen. »Voller Hunger, wirtschaftlichem Elend und Enttäuschungen«, so formulierte es etwa William Clayton, der Unterstaatssekretär für Wirtschaftsfragen im State Department in einer Note vom 5. März 1947 an die Spitzen der Administration, stünden die meisten der europäischen Länder "hart am Abgrund und können jederzeit heruntergestoßen werden; andere sind schwer bedroht." In Griechenland und Frankreich sei abzusehen, wie auf den wirtschaftlichen Zusammenbruch kommunistische Machtübernahmen folgen würden; ohne amerikanische Hilfe größten Ausmaßes "wird sich die Lage so hoffnungslos verschlimmern, dass sie zwangsläufig zum Dritten Weltkrieg führen wird. «8

Das Resultat dieser Befürchtungen waren erstens die Truman-Doktrin vom 12. März 1947, die allen »freien Völkern« amerikanische Unterstützung beim Widerstand gegen »Unterwerfung durch bewaffnete Minderheiten oder durch Druck von außen" zusicherte ${ }^{9}$ und zweitens die Ankündigung des Marshall-Plans am 5. Juni 1947, die den Europäern wirtschaftliche Unterstützung bei gemeinsamen Bemühungen um den Wiederaufbau in Aussicht stellte. Subventionen und Darlehen sollten Engpässe beim Wiederaufbau beseitigen; dazu sollten die Produkti-

7 Harris, Seymour E.: The European Recovery Program, Cambridge, Mass. 1948, S. 30 f., 40, 1- 51, 92, 168f., 249, $252-259$.

8 Zitiert bei Clayton-Garwood, Ellen: Will Clayton. A Short Biography, Austin 1958, S. 115 - 118,

9 "Rede des amerikanischen Außenministers, George C. Marshall, vor der Havard-Universität zur Hilfeleistung für die europäischen Länder (Marshall Plan)« in: Europa-Archiv 2 (1947), S. 819 -821. 
onsbeschränkungen, die man dem besiegten Deutschland aus Sicherheitsgründen auferlegt hatte, weitgehend aufgehoben werden, und die Handelsschranken zwischen den beteiligten Ländern sollten so schnell wie möglich beseitigt werden. Das lief auf eine wirtschaftliche Integration der europäischen Teilnehmerländer hinaus; und wenn es nicht gelang, die sowjetische Besatzungszone Deutschlands in das Programm einzubeziehen, war damit auch die Errichtung eines westdeutschen Staates vorprogrammiert. ${ }^{10}$

\section{Sowjetische Abwehrpläne}

In Moskau hat man die Chancen für kommunistische Machtergreifungen im westlichen Europa viel geringer angesehen als in Washington. "Dieser Krieg ist nicht wie in der Vergangenheit", hatte Stalin einer Delegation der jugoslawischen Parteiführung im April 1945 erläutert. »Wer immer ein Gebiet besetzt, erlegt ihm auch sein eigenes gesellschaftliches System auf. Jeder führt sein eigenes System ein, soweit seine Armee vordringen kann. Es kann ja nicht anders sein. «11 Entsprechend hatte er die Kommunisten in Frankreich in realistischer Einschätzung der Machtverhältnisse angewiesen, die Widerstandsbewegung in eine "Bewegung zum Wiederaufbau eines starken Frankreichs und zur Stärkung der Demokratie» zu überführen. "In die Plattform dieser Bewegung muss in erster Linie der wirtschaftliche Aufbau des Landes und die Festigung der Demokratie gehören. « ${ }^{12}$ Für die kommunistischen Parteien in Italien und in den kleineren westeuropäischen Ländern galten die gleichen Vorgaben. Der Aufstandsbewegung in Griechenland gegen das autoritäre Athener Regime, die von den griechischen Kommunisten wesentlich mitgetragen wurde, verweigerte Stalin jede Unterstützung. ${ }^{13}$

Im Hinblick auf das besiegte Deutschland betrachtete er die "Vollendung der bürgerlich-demokratischen Revolution«, die 1848/49 stecken geblieben war, als Tagesaufgabe, und diese Aufgabe war nach seinem Verständnis nur gemeinsam mit den westlichen Besatzungsmächten zu verwirklichen. „Einheit Deutschlands sichern«, wies er darum die Führer der deutschen Kommunisten in einer Unterre-

10 Vgl. Hogan, Michael J.: The Marshall Plan. America, Britain, and the reconstruction of Western Europe, 1947-1952, Cambridge/ Mass. 1987.

11 Djilas, Milovan: Gespräche mit Stalin, Frankfurt a. M. 1962, S. 146.

12 Narinskij, Mikhail M.: I. V. Stalin i M. Torez, 1944-1947gg. Novie materiali, in: Novaja i nowejschaja istorija 7 (1996), Nr. 1, S. 18 -30; die Zitate S. 23, 25. Vgl. Loth, Wilfried: Die französischen Kommunisten und der Beginn des Kalten Krieges, in: Vierteljahrshefte für Zeitgeschichte 26 (1978), S. 7-65.

13 Woodhouse, Christopher F.: The Struggle for Greece, 1941-1949, London 1976. 
dung am 4. Juni 1945 an. ${ }^{14}$ „Die Besatzungsmächte kämen nach Deutschland, um den Faschismus und Militarismus auszurotten und die notwendigen Maßnahmen für eine demokratische Wiedergeburt des deutschen Volkes zu treffen«, so die Instruktion für die Mitglieder der "Gruppe Ulbricht", die den sowjetischen Besatzungstruppen in Deutschland zur Hand gehen sollte. »Es käme darauf an, unter peinlichster Beachtung der alliierten Vorschriften bei diesen Reformen aktiv mitzuarbeiten und für ihre konsequente Durchführung zu sorgen.«"15 Weit davon entfernt, auf einen raschen Abzug der amerikanischen Besatzungsmacht aus Deutschland zu spekulieren, wie man auf der westlichen Seite häufig vermutet hat, sorgte sich Stalin, dass die Amerikaner zu früh abziehen könnten - »bevor die Hauptaufgabe der Besatzung - die Entmilitarisierung und Demokratisierung Deutschlands - vollendet sind", wie der sowjetische Botschafter in Washington Nikolai Nowikow in einem Memorandum schrieb, das er am 27. September 1946 an Außenminister Wjatscheslaw Molotow schickte. ${ }^{16}$

Ein solcher vorzeitiger Rückzug der amerikanischen Besatzungstruppen aus Deutschland würde »die Vorbedingungen für das Wiederaufleben eines deutschen Imperialismus schaffen«, fügte Nowikow erläuternd hinzu, und das sei Teil einer höchst gefährlichen antisowjetischen Strategie des »amerikanischen Monopolkapitals«: »Europa ist aus dem Krieg mit einer vollständig zerrütteten Wirtschaft hervorgegangen. [...] Diese Situation verschafft dem amerikanischen Monopolkapital Aussichten auf enormen Gütertransfer und Kapitalimport in diese Länder - ein Umstand, der es ihm erlauben wird, in ihre nationalen Wirtschaften einzudringen.« Das Drängen der US-Regierung auf Einhaltung der Erklärung von Jalta nahm Nowikow als Versuch wahr, »den Einfluss der Sowjetunion auf ihre Nachbarländer zu begrenzen oder zu beseitigen." Dazu würden "reaktionäre Kräfte unterstützt», und es würde auch versucht, »Positionen für die Durchdringung ihrer Wirtschaften durch amerikanisches Kapital zu sichern."

Das Vordringen des amerikanischen Kapitals in West- wie in Osteuropa deutete er als Ausdruck eines Strebens nach Weltherrschaft, bei dem die Sowjetunion als "Haupthindernis» im Weg stünde. Die amerikanischen Imperialisten bereiteten darum auch einen »Krieg gegen die Sowjetunion« vor. Zwar könne zum gegenwärtigen Zeitpunkt niemand sagen, wann dieser Krieg stattfinden würde, doch

14 Notizen von Wilhelm Pieck zu dieser Unterredung in: Badstübner, Rolf/ Loth, Wilfried (Hrsg.): Wilhelm Pieck - Aufzeichnungen zur Deutschlandpolitik 1945 - 1953, Berlin 1994, S. 50 - 53.

15 Leonhard, Wolfgang: Die Revolution entlässt ihre Kinder, München 1969, S. 289.

16 Englische Übersetzung in: The Novikov Telegram, Washington September 27 1946, in: Diplomatic History 15 (1991), S. 527-537, hier S. 536. Hinweise, dass Stalin diese Sorge teilte, bei Loth, Wilfried: Stalins ungeliebtes Kind. Warum Moskau die DDR nicht wollte, Berlin 1994, S. 26 f. Weitere Zeugnisse für die Orientierung auf eine gemeinsame Verwaltung des besiegten Deutschlands ebd., S. 20 - 27. 
beabsichtigten die USA, ein imperialistisches Deutschland »in einem künftigen Krieg auf ihrer Seite zu nutzen. «17

Das Angebot des Marshall-Plans, das sich auch an die osteuropäischen Länder und die Sowjetunion richtete (aus ökonomischen Gründen ebenso wie aus taktischen Überlegungen), interpretierte Nowikow folgerichtig als Versuch einer »Blockierung der Demokratisierung der Länder Europas, der Stärkung antisowjetischer Kräfte» und der "wirtschaftlichen und politischen Unterjochung der europäischen Länder unter das amerikanische Kapital.« In einem Telegramm, das er am 24. Juni 1947 nach Moskau schickte, empfahl er, dass sich die Sowjetunion und die osteuropäischen Länder an dem Programm beteiligen sollten, um »die Verwirklichung der amerikanischen Pläne zur Unterjochung Europas und Schaffung eines antisowjetischen Blocks zu verhindern.» Die Instruktion für die Pariser Konferenz der Außenminister Großbritanniens, Frankreichs und der Sowjetunion, die Molotow am 25. Juni Stalin zur Billigung vorlegte, sah daraufhin vor, auf individuellen Hilfsanforderungen der europäischen Länder zu bestehen und eine Einbeziehung deutscher Ressourcen in das Wiederaufbauprogramm nur zuzulassen, wenn die westlichen Verbündeten endlich Zugeständnisse in der Frage deutscher Reparationsleistungen machten. ${ }^{18}$ Genauso verhielt sich Molotow, nachdem er am 27. Juni mit 89 Delegierten in Paris eingetroffen war.

Stalin hoffte also, dass die Regierungen Großbritanniens und Frankreichs die Gefahr einer Unterjochung der europäischen Länder durch den amerikanischen Imperialismus genauso hoch einschätzten wie er selbst, und dass sie darum dazu bewegt werden konnten, sich den amerikanischen Ambitionen zu widersetzen. Als der britische Außenminister Ernest Bevin und sein französischer Kollege Georges Bidault die sowjetischen Bedingungen für eine Beteiligung am MarshallPlan kategorisch ablehnten - sie fürchteten, dass eine sowjetische Beteiligung nur zu Komplikationen führen würde und der amerikanische Kongress die Hilfsgelder dann vielleicht gar nicht bewilligen würde -, setzte Stalin seine Hoffnungen auf die anderen europäischen Regierungen. Die Regierungen der osteuropäischen Länder wurden aufgefordert, an den Beratungen über das amerikanische Angebot teilzunehmen, zu denen Bevin und Bidault für den 12. Juli nach Paris eingeladen hatten. Sie sollten dort, »darlegen, dass der anglo-französische Plan unannehmbar ist, seine einstimmige Billigung verhindern und dann die Konferenz verlassen, dabei so viel Delegierte anderer Länder wie nur möglich mitneh-

17 Wie Anm. 16. Zur Entstehung des Memorandums vgl. Mal'kov, Viktor I.: Commentary, in: Diplomatic History 15 (1991), S. $554-558$.

18 Zitiert nach Parish, Scott: The Marshall Plan, Soviet-American-Relations, and the Division of Europe, in: The Establishment of Communist Regimes in Eastern Europe, 1944-1949, hrsg. von Norman Naimark/ Leonid Gibianskii, Boulder u. a. 1997, S. 267-290, hier S. 279-282. 
men«, so Molotow in gleichlautenden Botschaften an alle »befreundeten« Regierungen am 5. Juli. ${ }^{19}$

Aus diesem Plan wurde nichts, weil dem Sowjetdiktator wenig später Bedenken kamen, ob die westlichen Konferenzteilnehmer nicht eher die östlichen Delegierten auf ihre Seite ziehen würden. Entsprechend wurden die östlichen Regierungen am 8. Juli informiert, dass das Zentralkomitee der Kommunistischen Partei der Sowjetunion (KPdSU) "vorschlägt, eine Beteiligung an dem Treffen abzulehnen.«20 Die Regierungen des späteren Ostblocks folgten diesem »Vorschlag» manche allerdings erst, nachdem Moskau sie massiv unter Druck gesetzt hatte. Der tschechoslowakische Ministerpräsident Klement Gottwald und sein Außenminister Jan Masaryk reisten eigens nach Moskau, um doch noch eine Zustimmung zu einer Beteiligung ihres Landes an dem Programm zu erreichen. Dort wurde ihnen aber nur gesagt, dass die Sowjetregierung sofort den Freundschaftsvertrag von 1943 aufkündigen würde, wenn sie tatsächlich nach Paris fahren würden. Daraufhin sagten sie am 10. Juli ihre Beteiligung an der Konferenz ab, ebenso wie die ungarische Regierung. Tags zuvor hatten schon Jugoslawien und Bulgarien abgesagt, am 11. Juli folgten Rumänien, Albanien, Polen und selbst Finnland, dessen Regierung nach heftigen inneren Auseinandersetzungen einen Verzicht auf Hilfe der Zugehörigkeit zu einem von der Sowjetunion bekämpften Westblock vorzog. Damit war eine wesentliche neue Stufe der Einschränkung der Bewegungsfreiheit der osteuropäischen Länder erreicht und die Teilung des europäischen Kontinents entscheidend vertieft. Zu Recht urteilte Masaryk nach seiner Rückkehr aus der sowjetischen Hauptstadt: "Ich fuhr nach Moskau als der Außenminister eines souveränen Staates und kehre als Lakai der Sowjetregierung zurück. « ${ }^{21}$

Stalin hoffte nun, dass sich die westeuropäischen Regierungen in den Verhandlungen des in Paris eingesetzten "Committee of European Economic Cooperation" den hegemonialen Ambitionen der USA aus eigenem Antrieb widersetzen würden. Nachdem auch diese Hoffnung getrogen hatte, setzte er im September 1947 auf eine Mobilisierung der »demokratischen und patriotischen Kräfte» im Westen gegen den Marshall-Plan. ${ }^{22}$ Wenn sich schon die Regierungen von den amerikanischen Imperialisten hatten korrumpieren lassen, so sagte er sich nun, wür-

\footnotetext{
19 Veröffentlicht bei Takhnenko, Galina: Anatomy of a Political Decision: Notes on the Marshall Plan, in: International Affairs (Moskau), Juli 1992, S. 111-127, hier S. 123.

20 Ebd., S. 124.

21 Zitiert nach Lockhart, Robert B.: My Europe, London 1952, S. 125.

22 Stalin an Andrej Shdanow, zitiert bei Gibjanskij, Leonid Y.: Kak Woznik Kominform. Po novym archvnym materialam, in: Novaja i novejschaja istorija 4 (1993), S. 131-152.
} 
den sich doch die Nationen selbst nicht freiwillig der Herrschaft der USA und der Ausbeutung durch den amerikanischen Imperialismus unterordnen.

Auf der Gründungskonferenz des Kommunistischen Informationsbüros (Kominform) vom 22. bis 27. September 1947 im schlesischen Slarska Poreba (Schreiberhau) wurden die Führungen der kommunistischen Parteien Europas auf diese Mobilisierung eingeschworen. In seinem Einleitungsreferat, das Punkt für Punkt mit Stalin abgesprochen war, ${ }^{23}$ bekräftigte Parteisekretär Andrej Shdanow die Interpretation der amerikanischen Politik, die seit dem Herbst 1946 in Moskau kursierte: Die USA hätten sich zur »Hauptkraft des imperialistischen Lagers» entwickelt; dessen Hauptziel sei die »Festigung des Imperialismus, die Vorbereitung eines neuen imperialistischen Krieges, der Kampf gegen Sozialismus und Demokratie sowie die Unterstützung reaktionärer und antidemokratischer profaschistischer Regime und Bewegungen.« Sodann charakterisierte er den Marshall-Plan als Ausdruck des »aggressiven, unverhüllt expansionistischen Kurses«, den die amerikanische Politik nach dem Zweiten Weltkrieg eingeschlagen habe. Er sei ein "Plan zur Versklavung Europas«, dessen Wesen darin bestehe, »einen Block von Staaten, die durch Verpflichtungen den USA gegenüber verbunden sind, zusammenzuzimmern und die amerikanischen Anleihen als Gegenleistung für den Verzicht der europäischen Staaten auf ihre wirtschaftliche und später auch auf ihre politische Selbständigkeit zu gewähren. "Schließlich rief er die kommunistischen Parteien in West- wie in Osteuropa dazu auf, "sich an die Spitze des Widerstands gegen die Pläne der imperialistischen Aggression und Expansion auf allen Gebieten [zu] stellen [...] und alle demokratischen und patriotischen Kräfte des Volkes um sich sammeln. ${ }^{24}$ Mit »Streiks, Demonstrationen, politischem Streik, Massenmobilisierung", so die Notizen der jugoslawischen Delegierten in Slarska Poreba zu Shdanows Ausführungen, ${ }^{25}$ sollte dem Widerstand das nötige politische Gewicht verschafft werden.

Die Aussichten, dass der Marshall-Plan auf diese Weise zum Scheitern gebracht werden könnte, bezeichnete Shdanow als gut: „Wenn der Plan einer ıDawesierung، Europas sich seinerzeit als zum Scheitern verurteilt erwies [Shdanow spielte hier auf den Dawes-Plan von 1925 an], und zwar zu einer Zeit, wo die Kräfte des Widerstandes gegen den Dawes-Plan weitaus geringer als jetzt waren, so sind heute im Nachkriegseuropa, ganz zu schweigen von der Sowjetunion, durchaus genü-

23 Vgl. Wolkogonow, Dimitri: Stalin. Triumph und Tragödie, Düsseldorf 1989, S. 718.

24 Deutsche Übersetzung in Tägliche Rundschau 24.10.1947, hier zitiert nach Meissner, Boris (Hrsg.):

Das Ostpakt-System, Frankfurt a. M./ Berlin 1955, S. 89-97.

25 In einem Bericht an ihr Zentralkomitee vom 30.9.1947, veröffentlicht bei Dedijer, Vladimir: Novi prilozi za biografiju Jospia Broza Tita, Belgrad 1984, S. 275. 
gend Kräfte vorhanden, die diesen Knebelplan zum Scheitern bringen können. « ${ }^{26}$ Die jugoslawischen Delegierten notierten zu diesem Punkt: „Wenn nur zwei Millionen Menschen aufbrüllten, würden sie [gemeint waren die Franzosen] die Amerikaner und Engländer verjagen." Danach würde man wohl wieder in die französische Regierung zurückkehren können, an der man von 1944 bis Anfang Mai 1947 beteiligt gewesen war: "Später wird man sehen, ob irgendwelche Koalitionen möglich sind. «"27

Angesichts der neuen Aufgaben, die Stalin den Führern der kommunistischen Parteien damit zugewiesen hatte, ließen die Parteiführungen im westlichen Europa dem beträchtlichen sozialen Unmut freien Lauf, der sich durch die bisherige kommunistische Politik der Produktionssteigerung durch Konsumverzicht in den Reihen der Arbeiterschaft angesammelt hatte, und dem die Parteiführungen ohnehin kaum mehr widerstehen konnten. Die Streikbewegungen, die daraufhin ausbrachen, suchten sie dazu zu nutzen, den Marshall-Plan in den westeuropäischen Ländern innenpolitisch zu Fall zu bringen. In Frankreich begann die Streikwelle am 18. November 1947 und sie nahm bisweilen den Charakter eines allgemeinen Aufruhrs an: In der Tat waren es zwei Millionen Arbeiter, die in den Ausstand traten. Kohle wurde nicht mehr gefördert, die Lebensmittelversorgung wurde unterbrochen, auf Verkehrsverbindungen wurden Sabotageakte und Anschläge verübt. Im Parlament häuften sich die Tumulte. Einmal hielten kommunistische Abgeordnete die Tribüne eine ganze Nacht hindurch besetzt; der sozialistische Innenminister Jules Moch, Jude und Soldat beider Weltkriege, wurde mit "Heil Hitler!» niedergeschrien. In Marseille lieferten sich die Sicherheitskräfte und Streikende heftige Straßenschlachten. ${ }^{28}$ Am 9. Dezember brachen die Streiks allerdings zusammen. Konkrete Ergebnisse hatten sie bis dahin nicht gebracht, und es war auch nicht zu sehen, dass sich die Regierungsmehrheit davon beeindrucken lassen würde. In Italien dagegen, wo die Unruhen zur gleichen Zeit eingesetzt hatten, flackerten sie den ganzen Winter 1947/48 über immer wieder auf.

\section{Die Verhärtung der Fronten}

Das Ergebnis dieser spektakulären Streikaktionen - die die kommunistischen Parteiführungen nur zum Teil im Griff hatten - war allerdings keineswegs ein

\footnotetext{
26 Meissner, Ostpakt-System, S. 95.

27 Bericht vom 30.9.1947, zitiert nach Dedijer, Jospia Broza, S. 275.

28 Vgl. die eindrucksvolle Schilderung bei Fauvet, Jacques: Histoire du parti communiste français 1920 - 1976, Paris 1977, S. $393-400$.
} 
Scheitern des Marshall-Plans in Westeuropa, sondern im Gegenteil eine Konzentration aller nichtkommunistischen Kräfte auf den Aufbau Westeuropas im Rahmen des Marshall-Plans und die nahezu vollständige Isolierung der westlichen kommunistischen Parteien. Nicht nur in Frankreich, auch in Italien und in Belgien waren sie im Frühjahr 1947 aus den Regierungen ausgeschieden, doch hatte bislang kaum jemand damit gerechnet, dass sie auf Dauer in der Opposition bleiben würden. Insbesondere die meisten Sozialisten hatten eine baldige Rückkehr ihrer Bruderparteien in die Regierungsverantwortung gewünscht, und die Kommunisten hatten sich selbst noch ganz als verantwortliche Regierungsparteien gegeben, die den Marshall-Plan befürworteten. Erst das Spektakel der Novemberstreiks diskreditierte die kommunistischen Parteien in den Augen ihrer möglichen Koalitionspartner als bloße Werkzeuge Moskaus, die auf den Sturz der bestehenden Ordnung hinarbeiteten. Die Attacken auf den amerikanischen Imperialismus wurden als Angriffe auf das parlamentarisch-demokratische System interpretiert, alle antikommunistischen Ängste der Vergangenheit wurden wiederbelebt und die Kommunisten in das Getto ihrer "Gegenkultur» abgedrängt. Koalitionen mit kommunistischen Parteien galten fortan als grundsätzlich ausgeschlossen. Was die Sammlungsaufrufe eines Winston Churchill seit seiner Brandmarkung des "Eisernen Vorhangs« im März 1946 nicht vermocht hatten,,29 brachten nun die Aktionen der Kommunisten selbst zustande: Jetzt erst, um die Jahreswende 1947/48, wurde der Kalte Krieg in Europa zu einer innenpolitischen Realität.

Die Kominform-Propaganda und die anschließen Streikbewegungen ließen bei einem wachsenden Teil der europäischen Öffentlichkeit auch die Überzeugung entstehen, dass die Sowjetführung zur Verwirklichung ihrer Expansionspläne auch nicht vor dem Einsatz militärischer Mittel zurückschrecken würde. Am Rande der Londoner Tagung des Alliierten Außenministerrats vom 25. November - 15. Dezember 1947 - auf der Molotow vergeblich gegen die Entschlossenheit der drei westlichen Außenminister polemisierte, die westlichen Besatzungszonen Deutschlands in das europäische Wiederaufbauprogramm einzubeziehen - suchte Bevin, von Bidault nachdrücklich unterstützt, darum seinen amerikanischen Kollegen George C. Marshall für den Plan einer Allianz der Westmächte zu gewinnen, die insbesondere den Europäern einen militärischen Schutz durch die USA garantieren sollte: „Wir müssen eine Art westliches demokratisches System erfinden, das die Amerikaner, uns selbst, Frankreich, Italien, usw., und natürlich die Dominions umfasst, [...] kein förmliches Bündnis, sondern eine Verständigung

29 Churchill, Winston: Alliance of English-Speaking People: A shadow has fallen on Europe and Asia (Vital Speeches of the Day, Bd. 12, Nr. 11), S. $355-358$. 
auf der Grundlage von Macht, Geld und entschlossenem Handeln, [...] eine Art geistiger Föderation des Westens. ${ }^{30}$ Ein britisches Kabinettspapier vom 2. Januar 1948 hielt fest, dass auch Spanien und Deutschland, »ohne die kein westliches System vollständig sein kann«, in dieses Verteidigungssystem integriert werden sollten - »sobald die Umstände es erlauben. «¹

Marshall zeigte sich im Prinzip einverstanden, machte aber ein militärisches Engagement der USA in Westeuropa von einer vorherigen Intensivierung und Koordination der europäischen Verteidigungsanstrengungen abhängig; ohne eine solche Eigeninitiative, so meinte er, würde sich ein solches Engagement im Kongress noch viel weniger durchsetzen lassen als die Wirtschaftshilfe. Bevin und Bidault betrieben nun in aller Eile die Gründung eines Verteidigungspakts ihrer Länder mit den Benelux-Staaten als ersten Schritt zur Schaffung eines westlichen Bündnisses. Nach inoffiziellen Vorgesprächen Bevins mit Bidault und Churchill Ende Dezember in London proklamierte Bevin am 22. Januar 1948 in einer Unterhausrede die Idee einer „Western Union«. Am 4. März begannen die Paktverhandlungen, und schon am 17. März konnte der "Brüsseler Pakt» unterzeichnet werden, in dem sich Großbritannien, Frankreich, die Niederlande, Belgien und Luxemburg zu wechselseitiger Hilfe im Falle eines bewaffneten Angriffs in Europa verpflichteten sowie eine Intensivierung der politischen und wirtschaftlichen Zusammenarbeit zusagten. ${ }^{32}$

Die Hoffnungen auf ein Europa der »Dritten Kraft», das in den Spannungen zwischen den USA und der Sowjetunion vermittelte und so eine Konfrontation der Blöcke verhinderte, waren indessen Anfang 1948 immer noch so stark verbreitet, dass die Initiatoren des Brüsseler Paktes die tatsächliche Funktion des Bündnisses als Wegbereiter eines amerikanischen Militärschutzes und amerikanischer Militärhilfe für Europa so gut es ging verhüllten und es stattdessen als Grundlage für eine umfassende politische und militärische Zusammenarbeit Westeuropas im Sinne des Konzepts der »Dritten Kraft» präsentierten. Nur so schien es in den beteiligten Ländern innenpolitisch durchsetzbar zu sein. In Bevins Unterhausrede vom 22. Januar, die die öffentliche Diskussion um den Pakt bestimmte, war von sowjetischer Bedrohung nur verdeckt und von amerikanischem Schutz überhaupt nicht die Rede, dafür umso mehr von der »Konsolidierung" und "geistigen Union" Westeuropas und von seiner potentiellen Wirtschaftskraft zwischen den wanderen beiden großen Weltmächten, den

\footnotetext{
30 Foreign Relations of the United States 1947, Bd. 2, S. 815-817.

31 Zitiert bei Yergin, Daniel: Shattered Peace. The Origins of the Cold War and the National Security State, New York/ Boston 1977, S. 362 f.

32 Vgl. Varsori, Antonio: Il Patto di Bruxelles (1948). Tra integrazione europea e alleanza atlantica, Rom 1988.
} 
Vereinigten Staaten und der Sowjetunion.«33 Zumindest der belgische Außenminister Paul-Henri Spaak und die Sozialisten in der französischen Regierung wollten den Pakt in der Tat in diesem Sinne verwirklicht sehen, und auch bei Bevins Überlegungen spielte diese Perspektive eine Rolle. Es war daher zunächst keineswegs eindeutig abzusehen, wohin er tatsächlich führen würde - zu einer Blockbildung mit den USA oder zur Formierung eines relativ eigenständigen Europas.

Den entscheidenden Umschwung in der öffentlichen Meinung Westeuropas brachten erst die Nachrichten von der Ausschaltung der nichtkommunistischen Kräfte in der Tschechoslowakei Ende Februar 1948. Unter der Führung von Staatspräsident Eduard Benesch hatte die Tschechoslowakei ihre Freiheit bislang noch einigermaßen behaupten können, mit außenpolitischer Anpassung an die Sowjetunion und starker Beteiligung der Kommunisten an der Regierung. Angesichts einer bevorstehenden Wahlniederlage organisierte der kommunistische Partei- und Regierungschef Klement Gottwald nun aber Massenversammlungen in Prag, ließ am 21. Februar kommunistisch kontrollierte Polizeieinheiten in Stadt einmarschieren und ordnete am 24. Februar einen Generalstreik an. Dem Druck, der dadurch entstand, konnte sich Benesch nicht mehr widersetzen. Am 29. Februar akzeptierte er eine neue Regierung der "Nationalen Front», in der die bisherigen Positionen der Nichtkommunisten durch »zuverlässige» Vertrauensleute besetzt waren. Damit war der Weg zur Errichtung des kommunistischen Machtmonopols frei. $^{34}$

Dass Agitation, Demonstrationen und Streiks im Falle der Tschechoslowakei tatsächlich zu einer Machtergreifung der Kommunisten führten, bestärkte die Auffassung, dass die Aktionen im westlichen Europa das gleiche Ziel hatten. Nahezu alle Kommentatoren hielten nun die These vom notwendigerweise expansionistischen Charakter des Sowjetsystems für bestätigt. In der Bevölkerung wuchs die Furcht vor einer militärischen Aggression der Sowjetunion auf Westeuropa, und die verantwortlichen Politiker einschließlich der meisten bisherigen Verfechter der »Dritten Kraft» begannen, zumindest die Eventualität eines solchen Angriffs in ihr Kalkül einzubeziehen. Nur eine intellektuelle Minderheit der »DritteKraft»-Bewegung verfocht weiterhin einen "neutralen« Weg zwischen Ost und West. ${ }^{35}$ Die Mehrheit sah die westeuropäischen Demokratien wie die Väter der

33 Deutsche Übersetzung in: Jacobsen, Hans-Adolf (Hrsg.): Der Brüsseler Vertrag von 1948 und die durch ihn eingeleitete Entwicklung (Europa. Dokumente zur Frage der europäischen Einigung, Bd. 1), München 1962, S. 351-353.

34 Vgl. Myant, Martin: Socialism and Democracy in Czechoslovakia, 1945-1948, New York 1981, S. 167-218; Kaplan, Karel: Der kurze Marsch. Kommunistische Machtübernahme in der Tschechoslowakei 1945 -1948, München/ Wien 1981.

35 Vgl. für Frankreich Loth, Wilfried: Sozialismus und Internationalismus. Die französischen Sozialisten und die Nachkriegsordnung Europas 1940 - 1950, Stuttgart 1977, S. $188-201$. 
Eindämmungsdoktrin durch die Verbindung von subversiver Tätigkeit im Inneren und militärisch fundiertem Druck von außen bedroht; die Fähigkeit und Bereitschaft, zwischen kommunistischen Bewegungen und der Sowjetunion, kommunistischer Ideologie und realen sowjetischen Interessen zu differenzieren, nahm immer mehr ab. An der Notwendigkeit einer auch militärischen Allianz mit den USA bestand nun kein Zweifel mehr, auch wenn die meisten Verfechter der "Dritten Kraft« noch hofften, Europa innerhalb dieser Allianz eine relativ unabhängige Rolle sichern zu können. Bidaults wie Bevins inständige Bitten um amerikanischen Schutz fanden jetzt die notwendige innenpolitische Rückendeckung, sodass sich die Brüsseler Mächte schon einen Monat nach Vertragsabschluss offiziell um Unterstützung durch die USA bemühen konnten. ${ }^{36}$

Im Sinne einer "self fulfilling prophecy» wurde so der antikommunistische Westblock in Europa, gegen den sich die Kominform-Politik wandte, ab 1948 Realität. Zur Eskalation wechselseitiger Präventivmaßnahmen gegen die vermeintliche Bedrohung durch die Gegenseite trat die Eskalation feindseliger Rhetorik; wobei sich die westliche Freie-Welt-Ideologie defensiv gab, die Westmächte tatsächlich aber durchaus über Möglichkeiten verfügten, in den sowjetischen Sicherheitsbereich hinüber zu wirken, während sich die sowjetische Politik als Offensive gegen den »US-Imperialismus« aufführte, tatsächlich aber kaum Möglichkeiten hatte, in den Verhältnisse der westlichen Länder einzugreifen. Der OstWest-Konflikt wurde nun nicht mehr als bloßer Machtkampf zweier Weltmächte begriffen, in dem ein Ausgleich prinzipiell möglich war, sondern mehr und mehr als allumfassender Existenzkampf zweiter Gesellschaftssysteme, der nur mit Sieg oder Niederlage enden konnte. Es ist kein Zufall, dass jetzt, etwa von der Jahreswende $1947 / 48$ an, die Bezeichnung dieses Konflikts als »Kalter Krieg“ populär wurde.

36 Schreiben an die US-Regierung 17.4.1948 in Foreign Relations of the United States 1948, Bd. 3, S. 91. Zu den Motiven für eine Beteiligung an der atlantischen Allianz im Einzelnen Wiggershaus, Norbert/ Heinemann, Winfried (Hrsg.): Nationale Außen- und Bündnispolitik der NATO-Mitgliedsstaaten, München 2000. 
